\title{
Provider-initiated testing and counselling for HIV - from debate to implementation
}

\section{A policy space for expanded HIV testing}

Recent statements by the new Minister of Health about 'mass voluntary counselling and testing (VCT) campaigns', and references in the latest draft VCT policy to 'provider-initiated

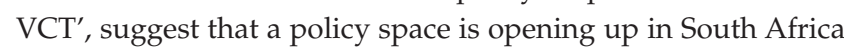
for the expansion of HIV testing models beyond the current VCT approach. The existing VCT programme is showing some successes. For example, the Human Sciences Research Council (HSRC) has reported a dramatic increase in the number of 15 48-year-olds who report awareness of their HIV status, from $11.9 \%$ in 2005 to $24.7 \%$ in $2008 .{ }^{1}$

Despite increased testing rates and willingness to test, most HIV-positive people do not know their status, do not consider themselves at risk and do not self-initiate testing, even with widespread awareness of and access to VCT services. ${ }^{2,3}$ Barriers associated with VCT uptake include clients having to initiate testing themselves, lengthy pre- and post-test counselling, implementation barriers (organisational management, supervision, human resource and infrastructure limitations), and patient concerns about confidentiality.,

Possible strategies for expanding HIV testing include 'provider-initiated HIV testing and counselling' (PITC) ${ }^{6}-$ also referred to as 'opt-out' HIV testing or 'routine offer of HIV testing' - and broad-based testing approaches such as 'mass VCT' campaigns, mobile VCT services, community- and homebased HIV testing initiatives and self-testing.

\section{Provider-initiated HIV testing and counselling: benefits and concerns}

In PITC, health providers offer HIV testing to all patients in medical settings, irrespective of their presenting complaint. Patients have the choice to opt out. If they choose to test, informed consent is confirmed verbally (written consent is only obtained if this is a requirement of the health authority) and the rapid test is done immediately. Lengthy pre-test counselling is not a requirement. Post-test counselling is focused on ensuring that patients understand the test result and prevention messages, helping them cope with the associated emotions, and making follow-up arrangements for clinical care, support and prevention services. ${ }^{6}$

The World Health Organization (WHO), the Joint United Nations Program on HIV/AIDS (UNAIDS) and the Centers for Disease Control (CDC) now recommend PITC as a streamlined and cost-effective HIV testing approach to rapidly and massively increase HIV testing rates in medical settings. ${ }^{6}$ The model has a number of advantages. PITC increases HIV testing rates across a range of high-, middle- and low-income settings with absolute increases varying from $5 \%$ to as high as $50 \%$ (baseline testing rates varied from $6 \%$ to $75 \%$ ). ${ }^{4,6,7}$ Patients also broadly find PITC acceptable, and sometimes preferable to VCT ${ }^{7,8}$ In South Africa, PITC addresses some health system barriers to testing such as the availability of counsellors and counselling rooms. Finally, PITC potentially normalises
HIV testing for patients and staff, by integrating testing and associated HIV care into standard clinical practice.

PITC can increase HIV testing rates in the South African setting. South African-controlled trials on PITC-type approaches showed significant increases in testing rates compared with VCT (14\% in tuberculosis patients ${ }^{9}$ and $13.8 \%$ in sexually transmitted infection (STI) patients ${ }^{10}$ ), and a quasiexperimental study showed increased HIV case detection among hospital outpatients. ${ }^{11}$

The PITC approach raises concerns around informed consent, counselling support, staff workload and access to care for the increased numbers who test positive. ${ }^{4,5}$ The main ethical concern is that testing, especially in resourceconstrained settings, will become coercive owing to unequal power relations between patients and providers. ${ }^{4,5}$ The PITC trial with STI patients in Cape Town showed that PITC was feasible to implement in a low resource-setting and that it could be implemented ethically. A proportion of patients who were offered the HIV test by nurses were able to decline testing ( $26.7 \%$ versus $13.5 \%$ for VCT), providing indirect evidence of patients exercising their right to decline testing, an indirect marker of the ethical implementation of PITC..$^{10}$ Qualitative investigation of informed consent practices found that nurses could apply the basic requirements of informed consent and that patients experienced the testing as voluntary and acceptable. $^{12}$

\section{Recommending PITC}

We therefore recommend PITC as an effective, ethical and acceptable method for increasing HIV testing rates in South Africa. However, PITC cannot be the only solution to expanding HIV testing, and there are challenges that will limit its impact and scope. Firstly, the increase in testing rates is not always dramatic. The absolute increases of around $14 \%$ (compared with VCT) in the two South African studies are significant but not sufficient to address low testing rates overall. Secondly, although the benefits of early diagnosis and early access to care are known, there are substantial gaps in access to care for those testing HIV positive. ${ }^{13}$ More effort is required to pro-actively link the increased numbers of HIV-positive patients diagnosed through PITC, and more monitoring is required to evaluate this. Finally, PITC implementation faces the same health systems barriers that confront VCT and all other clinical services (organisational, management and human resource difficulties).

\section{Focusing on the ethical implementation of PITC}

Maintaining the correct balance between 'normalising' HIV testing and ensuring its continued ethical application is most critical. PITC is not inherently coercive, and we found that it can be performed ethically in a low-resource, primary health care setting, but this does not mean that there is no risk of unethical testing behaviour (such as coercion), especially if 
PITC is up-scaled rapidly. ${ }^{4,5,8,10,12}$ Patients' rights are often not well protected in the South African public health service and patients are frequently not empowered to exercise their rights. For example, the PITC study among STI patients in Cape Town, where patients found the service to be both voluntary and acceptable, also showed that from an observer's perspective there was a spectrum in the ethical practice of nurses. For instance, some nurses were more explicit about and facilitative of the patient's right to refuse than others. ${ }^{12}$

The increasing normalisation of HIV testing - whether through PITC or other strategies - requires greater caution to ensure that patients' rights are protected, especially in settings where patient autonomy and participation in decision-making are not strongly embedded. However, debates around unethical practices and PITC should focus on implementation challenges rather than on the inherent qualities of PITC. The task is to balance the 'exceptionalising' impulses (e.g. where HIV testing is a parallel health service with exceptional requirements for test readiness and consent) and the 'normalising' impulses around HIV testing in a way that results in ethical, effective, efficient and sustainable services. The respect for patient

\section{Natalie Leon}

Health Systems Research Unit

Medical Research Council of South Africa

Cape Town

\section{Christopher J Colvin}

School of Public Health and Family Medicine University of Cape Town

\section{Simon Lewin}

Preventive and International Health Care Unit Norwegian Knowledge Centre for the Health Services Oslo, Norway, and

Health Systems Research Unit

Medical Research Council of South Africa

Cape Town

\section{Catherine Mathews}

Health Systems Research Unit

Medical Research Council of South Africa

Cape Town, and

School of Public Health and Family Medicine

University of Cape Town

\section{Karen Jennings}

HIV Director, City of Cape Town Health Department

Corresponding author: N Leon (natalie.leon@mrc.ac.za) autonomy that is a strong feature of the current VCT approach would be continued by strategies such as brief provider training on PITC that emphasise the importance of ensuring patient informed consent, together with maintaining the requirement for separate written consent. At the minimum, patients need to know the purpose and benefits of HIV testing and that they have the right to refuse testing, and health providers need to know how to motivate for HIV testing, to appreciate the importance of the patient's informed consent, and to convey a HIV-positive result in a supportive manner. Health providers will also need guidance on how to integrate HIV testing and follow-up care efficiently into routine care. If we use this pro-active and inclusive approach to HIV testing, service users (and service delivery) will benefit from increased HIV testing in medical settings and from the associated opportunities for increased and early linkage to HIV care, treatment, and prevention services.

Competing interest. The authors declare that they have no competing interest.

References

1. Shisana O, Rehle T, Simbayi LC et al. South African National HIV Prevalence, Incidence, Behavio and Communication Survey 2008: A Turning Tide amongst Teenagers? Cape Town: Human Sciences Research Council Press, 2008. http://www.mrc.ac.za/pressreleases/2009/sanat.pdf (accessed 27 November 2009).

2. WHO/UNAIDS/UNICEF. Towards Universal Access: Scaling Up Priority HIV/AIDS Interventions in the Health Sector. Progress Report. Geneva: WHO, 2007. http:/ / www.who.int/ hiv/pub/2008progressreport/en/ (accessed 19 October 2009).

3. Pettifor AE, Steffenson A, Hlongwa-Madikizela, McPhail C, Vermaak K, Kleinschmidt I. HIV and Sexual Behavior among Young South Africans: A National Survey of 15 Year Olds. Johannesburg: Reproductive Health Research Unit, University of Witwatersrand, 2004 http:/ / www.kff.org/southafrica/7066.cfm (accessed 27 November 2009).

4. Jürgens R.'Routinizing' HIV Testing in Low- and Middle-Income Countries - Background Paper. New York: Public Health Program of the Open Society Institute, 2006.

5. Nieburg P, Cannell T, Morrison JS. Expanded HIV Testing: Critical Gateway to HIV Treatment and Prevention Requires Major Resources, Effective Protections. Washington DC: Center for expandedhivtesting.pdf (accessed 14 September 2009).

6. WHO/UNAIDS. Guidance on Provider-Initiated HIV Testing and Counselling in Health Facilities. Geneva: World Health Organisation, 2007.

7. Chou R, Huffman LH, Fu R, Smits AK, Korthuis P. Screening for HIV. A review of the evidence for the US Preventive Services Task Force. Ann Intern Med 2005; 43: 55-73.

8. Weiser SD, Heisler M, Leiter K, et al. Routine HIV testing in Botswana: A population-based study on attitudes, practices and human rights concerns. PLoS Med 2006; 3: e216

9. Pope DS, DeLuca AN, Kali P, et al. A cluster-randomized trial of provider-initiated (opt-out) HIV counseling and testing of tuberculosis patients in South Africa. J Acquir Immune Defic Syndr 2008; 48: 190-195.

10. Leon N, Naidoo P, Mathews C, Lewin S, Lombard C. The impact of provider-initiated (optout) HIV testing and counseling of patients with sexually transmitted infection in Cape Town, South Africa: A controlled trial. BMC Implementation Science 2010; 5(8). http://www. implementationscience.com/content/5/1/8 (accessed 20 February 2010).

11. Bassett IV, Giddy J, Nkera J, et al. Routine voluntary HIV testing in Durban, South Africa: The experience from an outpatient department. J Acquir Immune Defic Syndr 2007; 46(2):181-186.

12. Leon N, Mathews C, Lewin S. Provider-initiated testing and counseling (PITC) and informed consent in a high HIV prevalence setting: Cape Town, South Africa. Poster presentation at the 11th World Congress Africa conference of the International Union Against Sexually Transmitted Infections, Cape Town, 9 - 12 November 2009.

13. Uphold R, Mkanta WN. Review: use of health care services among persons living with HIV Infection: state of the science and future directions. AIDS Patient Care STDS 2005; 19(8):473485 . 